\title{
Optimization Model of Cylindrical Resonant Cavity Slot Aiming on Radiation Reduction
}

\author{
Yang Liu ${ }^{1, a}$ \\ ${ }^{1}$ North China Electric Power University (Baoding). Huadian Road No. 689. Baoding City in \\ Hebei ,China \\ a835341518@qq.com
}

Keywords: Cylindrical resonant cavity, Slot radiation power, HFSS simulation, Gaussian function fitting, Linear constraint optimization model

\begin{abstract}
This paper mainly studies the optimization design method of annular slot in the cylindrical resonant cavity. Firstly, we illustrate the microwave resonant cavity perturbation method and radiation power's influence on the cavity; then using HFSS to simulate the radiation power calculation, in order to convenient facilitate results in optimal design, class quadratic Gaussian function is used to fit the data of the radiation power to get fitting equation between the slot width and slot position; Finally, we establish optimization model to the purpose of radiation power minimum based on the fitting function and using HFSS numerical simulation to verify the feasibility of the method.
\end{abstract}

\section{1、Brief Introduction of Microwave Resonant Cavity Perturbation Method}

The basic idea of microwave resonant cavity perturbation is the microwave resonant cavity resonance frequency will be offset with the variation of dielectric constant of dielectric cavity. When the resonant cavity semi closed, the current through the annular slot will out near field radiation, which will have an impact on the human body. In addition, the radiation will reduce the stored energy of the resonant cavity, causing unnecessary power loss; Radiation electromagnetic field will be generated on the main mode of resonant cavity interference patterns, disrupting main mode resonance, is not conducive to sweep observe and capture. In order to reduce electromagnetic radiation power of the slot, we design optimization model of the cylindrical resonant cavity slot.

\section{2、 Fitting Equation of the Slot Radiation Power}

Due to the calculation of cylindrical resonant cavity radiation power should take complicated boundary conditions into account, it is difficult to get exact analytic expressions, the method of complex numerical integration and matrix solution calculation is difficult to be used for the optimization model. To this end, we use HFSS electromagnetic simulation of microwave resonance cavity to simulate multiple position and width of slot, get a lot of data, and then the fitting equation of radiation power to slot position and width is obtained by using data, to prepare for the next step optimization model. Cylindrical cavity HFSS electromagnetic simulation is shown in Figure 1.

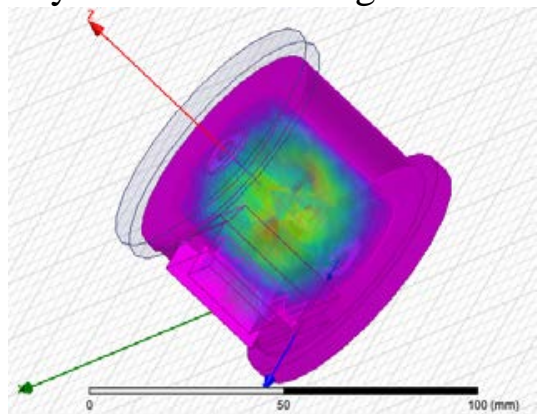

Fig. 1 A cylindrical cavity under HFSS simulation

We use HFSS established a size of single slit cavity 3D model, setting material, the boundary and 
the excitation frequency conditions. Then we numerically solve the radiation power in a cylindrical resonant cavity whose radius $R=2 \mathrm{~cm}$, the slot width in between $\Delta w=0.2 \mathrm{~cm} \sim 0.6 \mathrm{~cm}$ and slots in different position of cavity, computational results as shown in Figure 2.

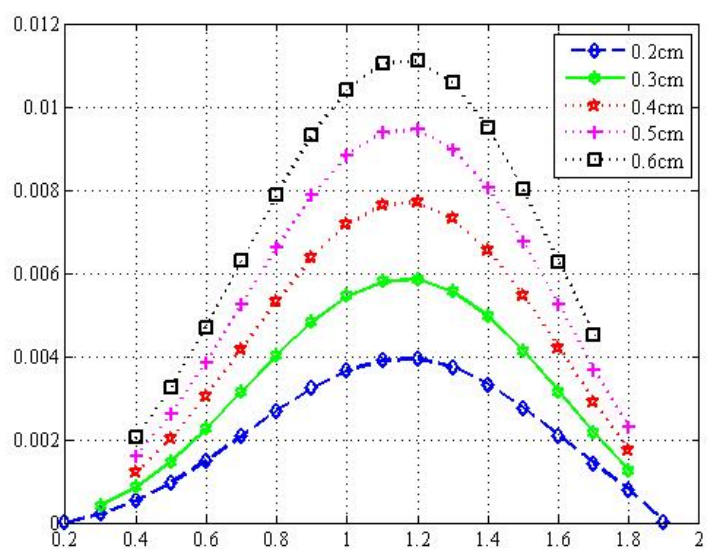

Fig. 2 Slot radiation power in different position and width

Among them, the $\mathrm{x}$ label represents the mid cycle radius to define the position of the slot. From the figure, the amount of slot radiation increases with the increase of the width, and with the relocation of the slot position power increased first and then decreased.

From the numerical results which the image shows, the radiation power distribution for different positions more satisfy the characteristics of Gaussian function and influence of slit width is mainly on the parameters of Gaussian function. To this end, we use MATLAB to fit quadratic Gaussian function and fit Gaussian function parameter with the slit width, the radiation power fitting relationship equation is below:

$$
\begin{aligned}
& P=0.016 \Delta w^{*} \exp \left(-\left(\frac{r-(1.23+0.03 \cos (16.24 \Delta w)+0.05 \sin (16.24 \Delta w))}{0.5044}\right)^{2}\right) \\
& +0.001 *(2+\cos (15.71 \Delta w)+\sin (15.71 \Delta w)) * \exp \left(-\left(\frac{r-(0.76+0.08 \cos (15.39 \Delta w)+0.06 \sin (15.39 \Delta w))}{0.3605}\right)^{2}\right)
\end{aligned}
$$

Among them, $P$ is the radiation power of the gap, $r$ is the radius of the mid circle, as the $\Delta w$ is slot width. By using fitting relation, we will simulation data into the radiation power of slot width and slot position binary relation.

\section{3、The Optimization Model of Cylindrical Cavity with Slot Width and Distribution}

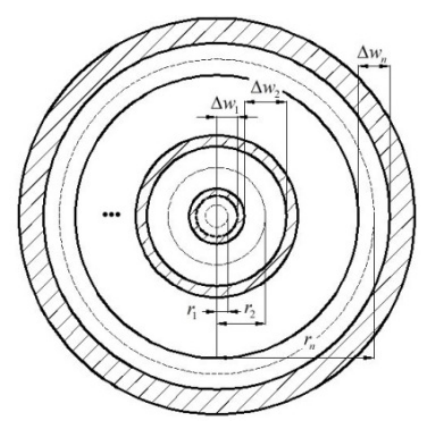

Fig. 3 Schematic diagram of slot parameters

Our optimization goal is to find an optimum aperture distribution and each slot width, for the total radiation power as much as possible small. Set slot number as $n$, the slot width as $\Delta w_{1} \ldots \Delta w_{n}$, the radius of the mid circle as $r_{1} \ldots r_{n}$ respectively, we can get a linear constrained optimization 
model:

$$
\begin{aligned}
& \min \sum_{i=1}^{n} f\left(r_{i}, \Delta w_{i}\right) \\
& \text { s.t. } \\
& \left\{\begin{array}{l}
r_{1}-\frac{\Delta w_{1}}{2}=0 \\
\delta_{\min } \leq\left(r_{i+1}-\frac{\Delta w_{i+1}}{2}\right)-\left(r_{i}-\frac{\Delta w_{i}}{2}\right) \leq \delta_{\text {max }} \\
r_{n}-\frac{\Delta w_{n}}{2}=R-\delta_{\text {outside }} \\
r_{1} \geq 2 m m, r_{j} \geq 1.5 \Delta w_{j}, j=2, \ldots, n
\end{array}\right.
\end{aligned}
$$

Among them, $f$ is the fitting relationship (1), $\delta_{\min }$ and $\delta_{\max }$ are the minimum and maximum widths of the metal ring between the slots under the requirements, $\delta_{\text {outside }}$ is the outer cavity wall thickness, $R$ is the total radius of the resonant cavity.

In the optimization model, the first and third constraints are respectively defined the boundary conditions of the inner and the outer slot; the second constraint defines each slot in the middle of boundary conditions, which are not overlapped each slot; the four constraint ensures the steam flow, prevent the influence on the measurement results.

\section{4、Optimization Model Solution and HFSS Numerical Simulation}

We use the optimization model above to optimize of a 3 Slot resonant cavity. The total radius $R$

\begin{tabular}{|c|c|c|c|}
\hline Slot & 1 & 2 & 3 \\
\hline Mid cycle radius $r$ & $1.41 \mathrm{~mm}$ & $7.665 \mathrm{~mm}$ & $15.055 \mathrm{~mm}$ \\
\hline Slot width $\Delta w$ & $2.82 \mathrm{~mm}$ & $5.33 \mathrm{~mm}$ & $4.11 \mathrm{~mm}$ \\
\hline Metal ring width & $2.18 \mathrm{~mm}$ & $2.67 \mathrm{~mm}$ & $2.89 \mathrm{~mm}$ \\
\hline
\end{tabular}
is $2 \mathrm{~cm}$. The $\delta_{\min }=1 \mathrm{~mm}, \delta_{\max }=3 \mathrm{~mm}$.

The solutions are as follows:

Table 1 Optimized solution of the slots

The total radiated power $\sum_{i=1}^{n} f\left(r_{i}, \Delta w_{i}\right)$ is 0.0117 watts. In order to validate the accuracy of the optimize slot calculation; we use HFSS electromagnetic numerical simulation software to simulate the situation.

According to the optimized size, draw the three-dimensional model of the cylindrical cavity, as shown in figure 4 . Then set up the material, boundary and excitation frequency. We calculated the electric field intensity and magnetic field intensity distribution of the cavity (Figure 5) and radiation parameters. Using HFSS calculation, the amount of gap radiation obtained is 0.0122 watts and the calculated values above is 0.0117 watts. We can think of the model can be used for the design of cylindrical resonant cavity in the slot. 


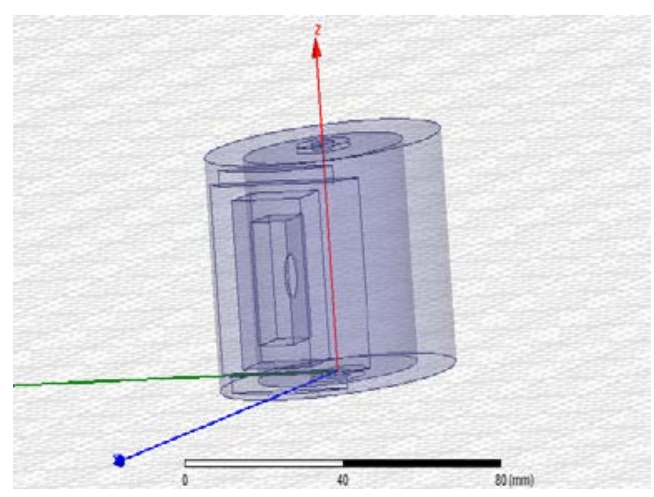

Fig. 4 Optimization model of three-dimensional cylindrical cavity
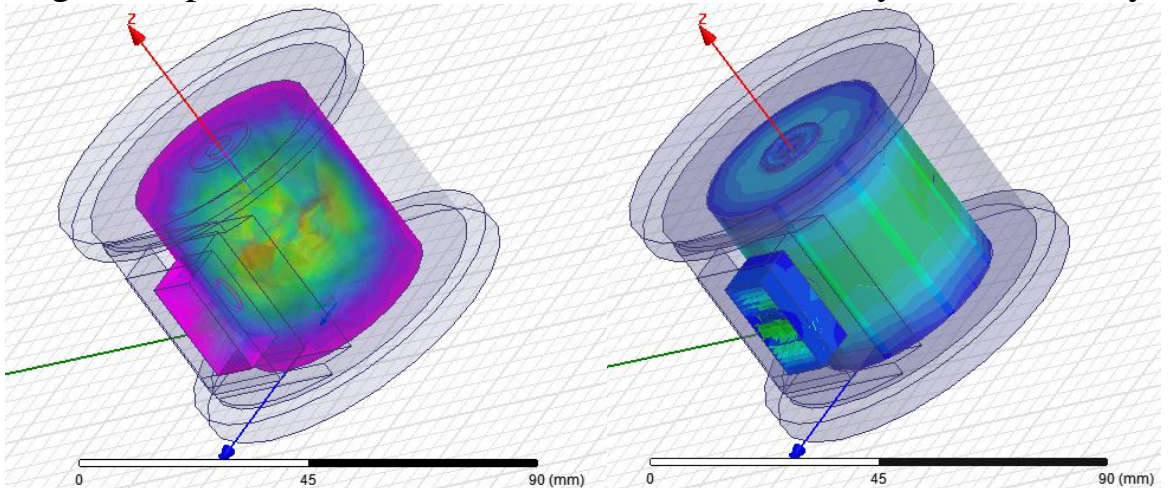

Fig. 5 The electric field intensity and magnetic field intensity distribution of cylindrical cavity

\section{Reference}

[1] Han Zhonghe, Zhang Shu'e, Yang Kun, steam turbine exhaust steam moisture resonator micro interference measurement [J]. Proceedings of the CSEE, 2002.

[2] Li Yanfeng, Wang Xinjun, and [J], analysis of steam turbine steam humidity measurement method of turbine technology, 2000, 42 (3): 156-161.

[3] Han Zhonghe, Qian Jiangbo. study on a method of steam Wetness measurement based on microwave resonant cavity [C]. The Ninth International Conference on Electronic Measurement \&amp; Instrument. 2009: 1604-1607.

[4] Yang Kun, Han Zhonghe, research status quo of turbine steam wetness in Journal of North China Electric Power University[J], 2002

[5] Qian Jiangbo, the steam humidity measurement method and experimental research of system design, master's degree thesis of North China Electric Power University [D], 2004

[6] Han Zhonghe, steam turbine exhaust steam wetness with microwave resonant cavity measurement technology [D]. North China Electric Power University, PhD thesis, 2006 